\title{
CORRIGENDUM
}

\section{SYMPLECTIC FILLINGS OF LINKS OF QUOTIENT SURFACE SINGULARITIES - CORRIGENDUM}

\author{
MOHAN BHUPAL AND KAORU ONO
}

First published online by Duke University Press 1 September 2012, subsequently published online by Cambridge University Press 11 January 2016, doi:10.1017/S0027763000022297

D. Shin informed us of his joint work [3] and that one case is missing in our list in [2]. We claimed that the link of the quotient surface singularity $I_{30(5-2)+23}$ has four minimal symplectic fillings; however, there is a fifth one. Recall that in [2] we showed that the compactifying divisor of a tetrahedral, octahedral or icosahedral singularity of Type $(3,1)$ can be chosen to have the form indicated in Figure 1, where $D$ is a rational cuspidal curve, $A$ and $B$ are rational curves meeting $D$ at the cusp point and $C_{1}, \ldots, C_{k}$ are rational curves. The missing filling is a Case II filling. In [2], we represented these using the notation $\left(m ; D \cdot D,-c_{1}, \ldots,-c_{k} ; i, j ; a_{1} \times i_{1}, \ldots, a_{l} \times i_{l}\right)$, where $m$ denotes the index of the singularity and $-c_{1}, \ldots,-c_{k}$ denote the self-intersection numbers of the curves $C_{1}, \ldots, C_{k}$. This notation is meant to indicate that if we glue the corresponding symplectic filling $X$ to a regular neighborhood of the compactifying divisor, then there will be a pair of $(-1)$ curves, one intersecting $B$ and $C_{i}$ and one intersecting $D$ and $C_{j}$. In addition, there will be a further $a_{i}(-1)$-curves intersecting $C_{i}$ for each $i$. In this notation, the missing filling can be represented by

$$
(30(5-2)+23 ; 5,-2,-2,-3,-3 ; 4,4 ; 2) .
$$

We would like to point out that this omission in our original list was not due to a theoretical error. Rather, it was due to our overlooking of a case - the original list was prepared by hand using the restrictions given in Propositions 4.5, 4.8 and 4.10. The omission arose by our not considering all possibilities arising from Proposition 4.10 in the case of the link of the singularity $I_{30(5-2)+23}$. It has been verified by the first author using

2010 Mathematics subject classification. Primary 53D35; Secondary 57R17, 32S25.

(C) 2016 by The Editorial Board of the Nagoya Mathematical Journal 


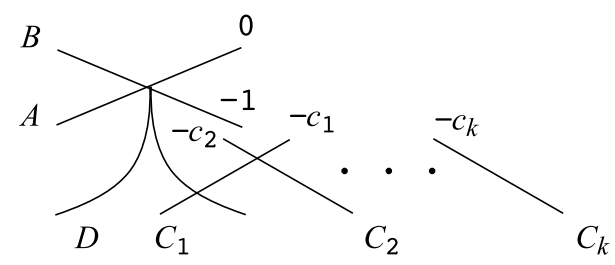

Figure 1.

Compactifying divisor for a tetrahedral, octahedral, or icosahedral singularity of Type $(3,1)$.

a program in $\mathrm{C}++[1]$ that this is the only case missing in our original list. Details of the algorithm found in the program in the case of Type $(3,1)$ singularities follow. The algorithm for the case of Type $(3,2)$ singularities is similar and the details are omitted.

\section{Algorithm for finding symplectic fillings of links of quotient surface singularities of Type $(3,1)$}

As mentioned above, corresponding to each link of a quotient surface singularity of Type $(3,1)$ there is a standard compactifying divisor $K$ consisting of a cuspidal rational curve $D$, a string of rational curves $C_{1}, \ldots, C_{k}$ and two more rational curves $A$ and $B$. For our purposes it is sufficient to record the self-intersection numbers $d=D \cdot D,-c_{1}=C_{1} \cdot C_{1}, \ldots,-c_{k}=$ $C_{k} \cdot C_{k}$. We will write $Q=Q_{K}=\left(d,-c_{1}, \ldots,-c_{k}\right)$. We handle Case I and Case II symplectic fillings of the singularity link associated with the data $Q$ separately. For Case I fillings, by Proposition 4.8, it is sufficient to list the numerical data associated to all preadmissible configurations $A^{\prime} \cup B^{\prime} \cup D^{\prime} \cup C_{1}^{\prime} \cup \cdots \cup C_{k}^{\prime}$ such that $D^{\prime} \cdot D^{\prime}=D \cdot D$ and $C_{i}^{\prime} \cdot C_{i}^{\prime} \geqslant C_{i} \cdot C_{i}$ for all $i$. For Case II fillings, by assumption and Proposition 4.10, first we have to identify the curves $C_{i}$ and $C_{j}$ such that there are exceptional curves $E$ and $F$ intersecting $B$ and $C_{i}$ and $D$ and $C_{j}$, respectively, in some rational surface $R$ containing the compactifying divisor $K$. Blowing down the curves $E$ and $F$ and denoting the resulting configuration $A^{\prime} \cup$ $B^{\prime} \cup D^{\prime} \cup C_{1}^{\prime} \cup \cdots \cup C_{k}^{\prime}$, by Proposition 4.10, it is then sufficient to find all preadmissible configurations $A^{\prime \prime} \cup B^{\prime \prime} \cup D^{\prime \prime} \cup C_{1}^{\prime \prime} \cup \cdots \cup C_{k}^{\prime \prime}$ such that $D^{\prime \prime} \cdot D^{\prime \prime}=D^{\prime} \cdot D^{\prime}$ and $C_{i}^{\prime \prime} \cdot C_{i}^{\prime \prime} \geqslant C_{i}^{\prime} \cdot C_{i}^{\prime}$ for all $i$. (Note that in [2], we did not define preadmissible configurations for Case II symplectic fillings of links of tetrahedral, octahedral or icosahedral singularities of Type $(3,1)$, however a definition similar to the other cases can be given.) 
$\overline{\text { Algorithm } 1 \text { Enumerate symplectic fillings of the link of a quotient surface }}$ singularity of Type $(3,1)$

procedure ListExceptionalCollections $(Q) \triangleright$ Given the data $Q=$ $\left(d,-c_{1}, \ldots,-c_{k}\right)$ of a compactifying divisor, list the set of all possible collections $S=\left(e_{1}, \ldots, e_{k}\right)$ that may arise as the data of a collection of exceptional curves

start with the empty collection $S_{0}=(0, \ldots, 0)$

increment $e_{1}$ in steps of 1 until it reaches $c_{1}-1$

then set $e_{1}$ to be 0 and increment $e_{2}$ by 1

then increment $e_{1}$ in steps of 1 again until it reaches $c_{1}-1$ again

then set $e_{1}$ to be 0 again and increment $e_{2}$ by 1 again

repeat this process until $e_{2}$ reaches $c_{2}-1$

then set $e_{2}$ to be 0 and increment $e_{3}$ by 1

repeat in this way until $S$ becomes $\left(c_{1}-1, \ldots, c_{k}-1\right)$

\section{end procedure}

procedure BlowDownCAseI $(Q, S) \triangleright$ Given the data $Q$ of a compactifying divisor $K$ of a Case I filling of the link of a quotient surface singularity of Type $(3,1)$ and exceptional curves data $S$, returns the data of the configuration $K^{\prime}$ obtained after a collection of exceptional curves represented by $S$ has been blown down

$Q \leftarrow\left(d,-c_{1}+e_{1}, \ldots,-c_{k}+e_{k}\right)$

return $Q$

end procedure

procedure AdmissibilityTestCaseI $(Q)$ D Check whether $Q=$ $\left(d,-c_{1}, \ldots,-c_{k}\right)$ is the data of a preadmissible configuration of a Case $I$ filling of the link of a quotient surface singularity of Type $(3,1)$

if $k=0$ then

if $d<9$ then

return 1

else

return 0

end if

end if

let $1 \leqslant s \leqslant k$ be the smallest index, if it exists, such that $c_{s}=1$

if no such $s$ exists then

return 0

end if 


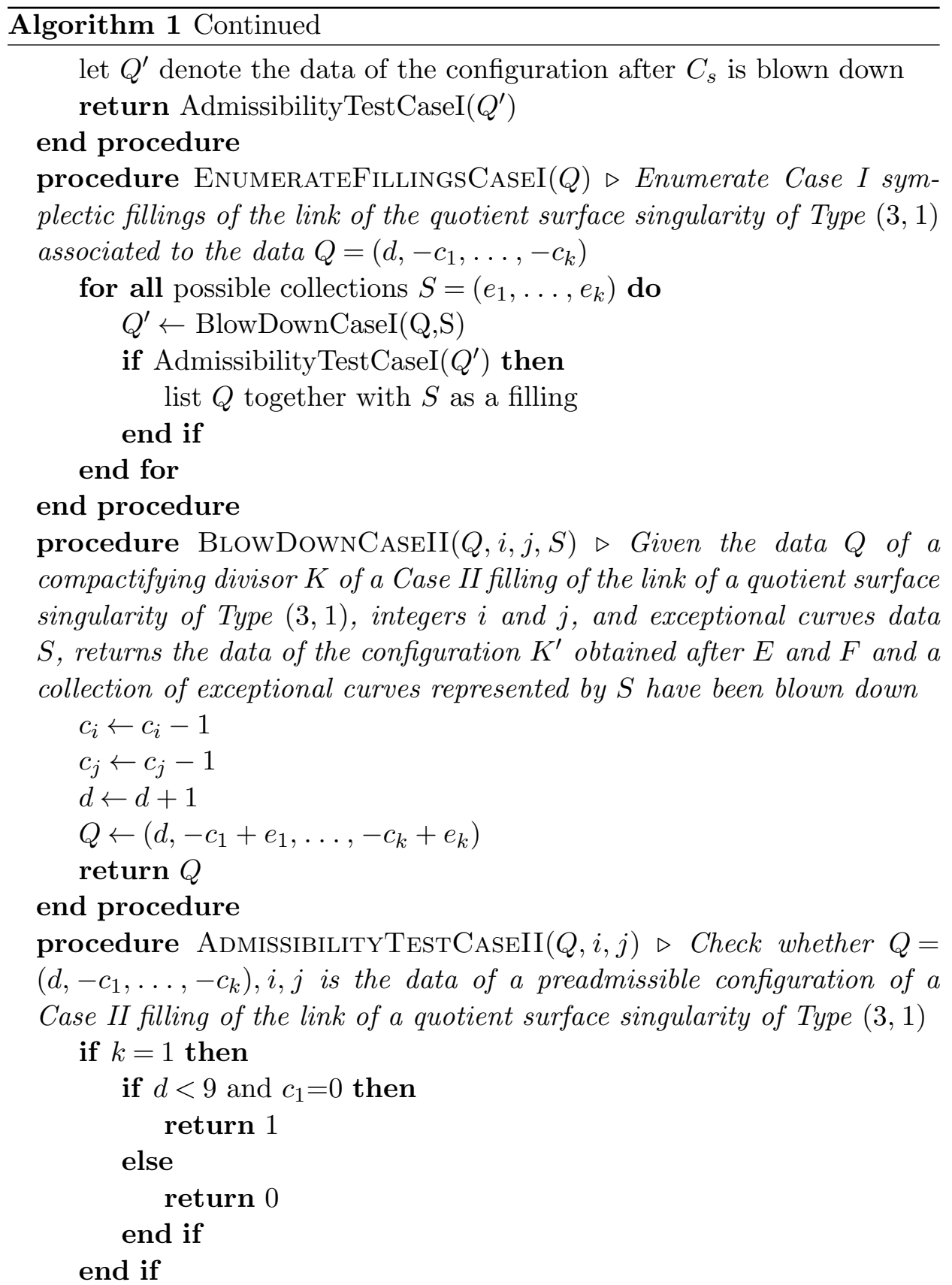

let $1 \leqslant s \leqslant k$ be the smallest index, if it exists, such that $c_{s}=1$ and $s \neq i$ and either $s \neq j$ or $s=k$ 


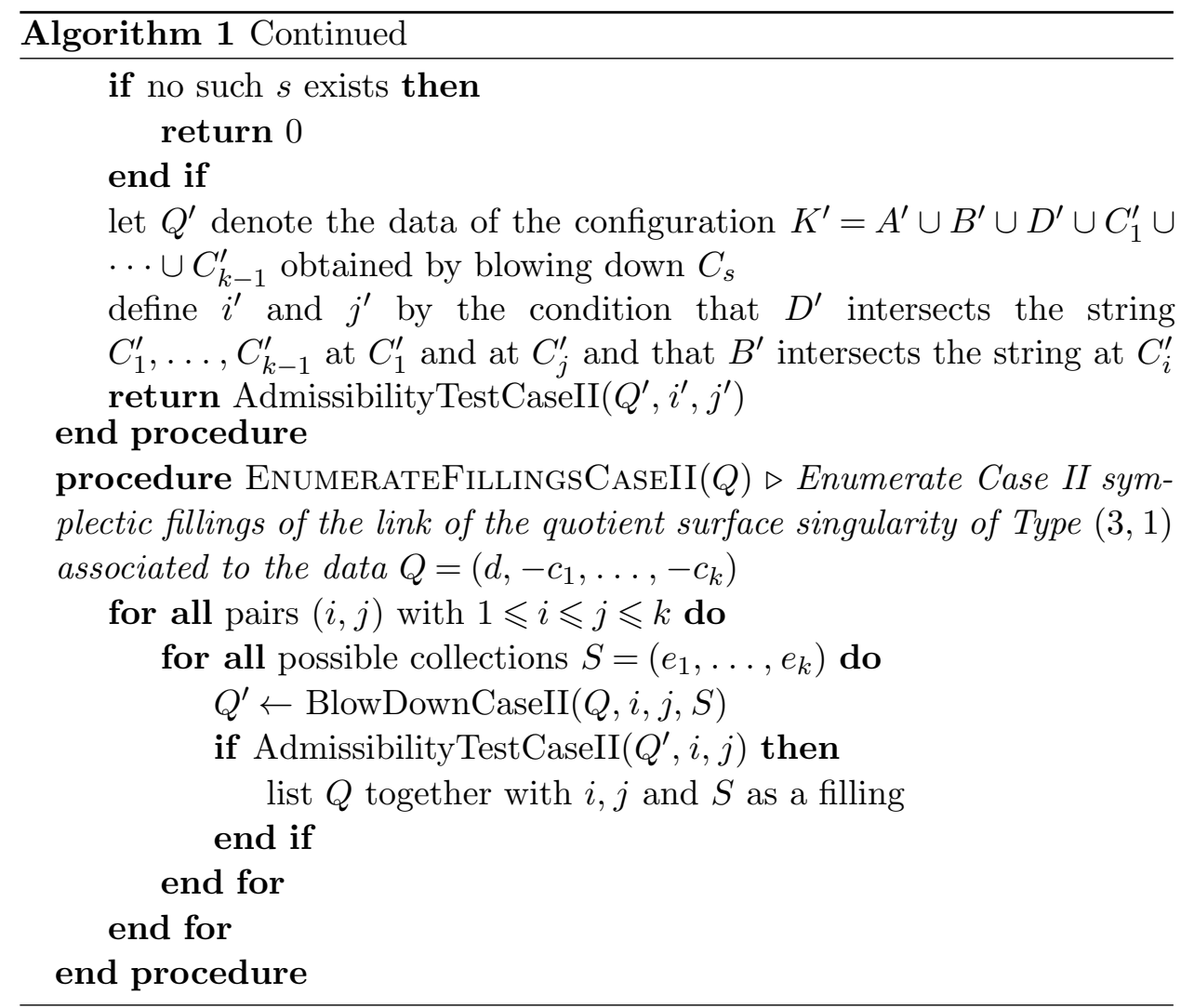

In the algorithm above, we assume that the compactifying divisor $K$ associated with the data $Q$ sits in some rational surface $R$. Collections of exceptional curves $\left\{E_{\alpha}\right\}$ in $R$ that are disjoint from $D \cup A \cup B$ and such that $E_{\alpha} \cdot \cup C_{i}=1$ for each $\alpha$ will be recorded by the data $S=\left(e_{1}, \ldots, e_{k}\right)$, where $e_{i}$ denotes the number of exceptional curves intersecting $C_{i}$. Thus to list all possible Case I fillings of the singularity link associated to $Q$, it is sufficient to enumerate all possible $k$-tuples $S$ such that on blowing down a collection of exceptional curves represented by $S, K$ is transformed into a preadmissible configuration. Similarly, to list all possible Case II fillings of the singularity link associated to $Q$, it is sufficient to list all possible numerical data $(i, j)$ and $S$ such that on blowing down $E$ and $F$ and a collection of exceptional curves represented by $S, K$ is transformed into a preadmissible configuration. 
Acknowledgments. We thank Dongsoo Shin and his collaborators for identifying the missing case in our list.

\section{Supplementary material}

Supplementary material is available at http://dx.doi.org/10.1017/nmj.2 016.42 .

\section{REFERENCES}

[1] M. Bhupal, C++ program to compute symplectic fillings of links of quotient surface singularities, Available at www.metu.edu.tr/ bhupal.

[2] M. Bhupal and K. Ono, Symplectic fillings of links of quotient surface singularities, Nagoya Math. J. 207 (2012), 1-45.

[3] H. Park, J. Park, D. Shin and G. Urzúa, Milnor fibers and symplectic fillings of quotient surface singularities, preprint 2015.

Mohan Bhupal

Department of Mathematics

Middle East Technical University

06800 Ankara

Turkey

bhupal@metu.edu.tr

Kaoru Ono

Research Institute for Mathematical Sciences

Kyoto University

Kyoto 606-8502

Japan

ono@kurims.kyoto-u.ac.jp 